\title{
Utilização da tomografia computadorizada de feixe cônico na obtenção de índices radiomorfométricos - Revisão de Literatura
}

\begin{abstract}
- Daniela Miranda Richarte de Andrade Salgado Departamento de Estomatologia da Faculdade de Odontologia, Universidade de São PauloSão Paulo, SP, Brasil • Jéssica Rabelo Mina Zambrana Departamento de Estomatologia da Faculdade de Odontologia, Universidade de São Paulo, São Paulo, SP, Brasil Nataly Rabelo Mina Zambrana Departamento de Estomatologia da Faculdade de Odontologia, Universidade de São Paulo, São Paulo, SP, Brasil • Rodrigo Alves Ribeiro Departamento de Estomatologia da Faculdade de Odontologia, Universidade de São Paulo, São Paulo, SP, Brasil • Bruno Vieira Caputo Departamento de Estomatologia da Faculdade de Odontologia, Universidade de São Paulo, São Paulo, SP, Brasil • Gilberto Araújo Noro-Filho Departamento de Estomatologia da Faculdade de Odontologia, Universidade de São Paulo, São Paulo, SP, Brasil - Claudio Costa Departamento de Estomatologia da Faculdade de Odontologia, Universidade de São Paulo, São Paulo, SP, Brasil
\end{abstract}

RESUMO | Os diferentes índices quantitativos e qualitativos que são utilizados para mensurar a qualidade óssea em radiografia panorâmica são denominados índices radiomorfométricos. Esses índices são propostos como ferramentas de rastreio da baixa densidade mineral óssea e da osteoporose, sendo considerados como métodos alternativos. Atualmente, pesquisadores têm utilizado esses índices em tomografias computadorizadas de feixe cônico (TCFC) com o intuito de verificar se esse exame também pode ser utilizado para busca de pacientes com baixa densidade mineral óssea. O objetivo desse estudo foi fazer uma revisão de literatura a respeito do uso da TCFC para a obtenção dos índices. Foram selecionados trabalhos que abordaram o uso da TCFC e índices radiomorfométricos para análise da qualidade óssea. Conclui-se que os índices radiomorfométricos podem ser obtidos em exames de TCFC, porém mais estudos são necessários devido à variabilidade de metodologias e parâmetros.

DESCRITORES | Tomografia Computadorizada de Feixe Cônico; Índices; Densidade Óssea.

ABSTRACT | Use of cone beam computed tomography in obtaining radiomorphometric indices - Literature Review • The different quantitative and qualitative indices used to measure bone quality in panoramic radiograph are known as radiomorphometric indices. These indices have been proposed as useful tools to screen for low bone mineral density and osteoporosis, being considered as alternative methods. Nowadays, researchers have been using these indices in cone beam computed tomography (CBCT) aiming to verify that this examination can also be used to search for patients with low bone mineral density. The aim of this study was to make a review of literature regarding the use of $\mathrm{CBCT}$ for obtaining the indices. Selected works which focused on the use of CBCT and radiomorphometric indices for bone quality analysis. We concluded that the radiomorphometric indices can be obtained in CBCT with software assistance, although more studies are necessary due to the variability methodologies and parameters variability.

DESCRIPTORS | Cone Beam Computed Tomography; Indices; Bone density;

AUTOR CORRESPONDENTE | - Daniela Miranda Richarte de Andrade Salgado Departamento de Estomatologia da Faculdade de Odontologia, Universidade de São Paulo, São Paulo, SP, Brasil • Av. Professor Lineu Prestes, 2227 São Paulo, SP, Brazil • 05508-000 E-mail daniricharte@usp.br

- Received Apr 08,2015 • Accepted Jun 02,2015

- DOI http://dx.doi.org/10.11606/issn.2357-8041.clrd.2015.119655 


\section{INTRODUÇÃO}

A osteoporose é considerada uma doença sistêmica, progressiva, caracterizada pela diminuição da massa óssea, resultado da deterioração da microarquitetura do osso, que ocasiona fragilidade e aumenta o risco de fratura. ${ }^{1}$ É uma doença considerada silenciosa, porque muitas de suas alterações são encontradas apenas quando ocorrem fraturas ósseas, o que demonstra a importância do diagnóstico antecipado. ${ }^{2}$

O rastreamento da osteoporose ${ }^{3}$ pode ser realizado pelos cirurgiões dentistas por meio de radiografias panorâmicas, comumente utilizadas nas avaliações iniciais dos pacientes, e também pela aplicação de índices radiomorfométricos, que permitem uma análise quantitativa e qualitativa da cortical mandibular.

Com a descoberta da osseointegração, os cirurgiões dentistas passaram a se preocupar mais com a qualidade e quantidade óssea, e com isso cresceu a utilização de implantes na reabilitação oral.4,5 Assim, aumentou-se o número de solicitações dos exames de tomografia computadorizada de feixe cônico (TCFC) para avaliação desses casos.

A TCFC foi introduzida na odontologia em $1998^{6}$ e, nos últimos anos, vem substituindo a tomografia convencional no diagnóstico em odontologia7 - alcançando ampla aceitação em imagem dentomaxilofacial -, porque permite menor tempo de exame, menor dose de radiação e redução no custo. ${ }^{8}$

Existem estudos que utilizaram a TCFC como método de rastreamento da osteoporose, porém com metodologias bem diferentes. O objetivo deste estudo foi realizar uma revisão de literatura a respeito do uso da TCFC na obtenção de índices radiomorfométricos que permitem uma avaliação da qualidade óssea da mandíbula.

\section{REVISÃO DE LITERATURA}

A osteoporose, na maioria das vezes, inicia-se a partir dos cinquenta anos, e estima-se que uma em cada duas mulheres e um em cada quatro homens com mais de 50 anos terão uma fratura relacionada à osteoporose em suas vidas. ${ }^{3}$ Fatores como idade, história familiar de fratura, baixo índice de massa corpórea e fratura prévia por fragilidade são componentes para o risco de fraturas. ${ }^{9}$ Outros fatores de risco, que também colaboram para o desenvolvimento da osteoporose, são ingestão insuficiente de cálcio, falta de exercício físico e hábitos tóxicos, como tabagismo e consumo excessivo de álcool ou medicamentos - especialmente glicocorticoides. ${ }^{10}$

A deficiência de estrogênio é o principal fator de contribuição para a perda óssea após a menopausa. ${ }^{11}$ Além disso, essa deficiência afeta o metabolismo ósseo e causa alterações na densidade mineral óssea (DMO), afetando os ossos maxilares, assim como o restante do organismo. A DMO da mandíbula se correlaciona positivamente com DMO da coluna lombar e do fêmur, importantes sítios na análise da osteoporose, ${ }^{12}$ e o osso mandibular de indivíduos com osteoporose se apresenta como um osso cortical menos espesso e mais poroso. ${ }^{13}$

O diagnóstico da osteoporose é realizado pela avaliação da coluna lombar, fêmur proximal, colo femoral ou fêmur total e antebraço, de acordo com os critérios da Organização Mundial da Saúde (OMS). O exame padrão-ouro é a absorciometria de dupla energia de raios-X, conhecido como densitometria óssea (DEXA) que indica a situação atual do paciente. Essa técnica envolve a imagem digital para localizar as regiões do esqueleto de interesse, seguido por estimativa de atenuação de raios-X em cada região. ${ }^{14}$

Como a osteoporose é uma doença sistêmica que envolve todos os ossos do esqueleto, as radiografias permitem também a visualização dos ossos maxilares e das estruturas dentárias, e, com isso, podem oferecer uma oportunidade de ser uma ferramenta de rastreio das alterações ósseas decorrentes da osteoporose. ${ }^{15,16}$ 
A literatura mostra que outros métodos de análise da qualidade óssea foram propostos, utilizando os índices radiomorfométricos em radiografias panorâmicas. ${ }^{11,12,17-19}$ Atualmente, alguns pesquisadores $^{1,16,20,21}$ começaram a utilizar a TCFC como uma ferramenta de diagnóstico prévio das alterações ósseas na mandíbula.

Scarfe et al. $^{22}$ relataram que a TCFC fornece imagens claras de estruturas altamente contrastantes e é extremamente útil para a avaliação óssea, embora existam limitações na utilização desta tecnologia para a visualização dos tecidos moles. Além disso, é uma técnica de aquisição de imagens médicas baseadas no feixe centrado de raio $\mathrm{X}$ com formato cônico em um detector bidimensional (2D). $\mathrm{O}$ conjunto fonte de raios $\mathrm{X}$ e receptor de imagens faz um movimento de rotação, em $360^{\circ}$, uma única vez, ao redor da região de interesse. As imagens são enviadas para o computador e são reconstruídas em um conjunto de dados tridimensionais (3D); desses dados podem ser obtidos cortes axiais, coronais e sagitais, e também reconstruções panorâmicas e cefalométricas a partir da imagem inicial. ${ }^{23,24}$

Na prática clínica, a qualidade da imagem de exames tomográficos em TCFC e a capacidade para mostrar características anatômicas e patológicas são influenciadas por um número de variáveis, tais como a unidade de digitalização, o campo de visão (FOV), objeto examinado, tempo de exame, tensão de tubo e amperagem, e também resolução espacial definida pelo tamanho do voxel. O tamanho de um voxel é definido por sua altura, largura e profundidade; voxels em TCFC são geralmente isotrópicos (os três parâmetros são iguais)., 75,26

Koh e Kim ${ }^{1}$ realizaram um estudo para avaliar o uso de índices de tomografia computadorizada, utilizando um tomógrafo de feixe cônico para verificar a densidade mineral óssea (DMO) em mulheres osteoporóticas na pós-menopausa. Os autores propuseram a utilização de um novo termo, o "ITC" (índice de tomografia computadorizada), para mensurar as medidas nos exames tomográficos e, com isso, concluíram que os índices de tomografia computadorizada podem ser utilizados para avaliar imagens de mulheres osteoporóticas.

No estudo de Diniz-Freitas et al., ${ }^{20}$ os autores avaliaram a largura da cortical mandibular (IM) em mulheres osteoporóticas que fazem uso de bifosfonatos orais. Os resultados mostraram que a altura da borda inferior da mandíbula ao forame mentual foi similar tanto no grupo das pacientes com osteoporose como no grupo controle. Porém, a largura da cortical mandibular foi significativamente diferente na comparação entre os dois grupos, o que levou os autores a concluírem que a largura da cortical mandibular foi maior em mulheres osteoporóticas tratadas com bifosfonatos do que em mulheres que não eram osteoporóticas.

Gomes et al. ${ }^{21}$ realizaram um estudo com o objetivo de comparar a avaliação do Índice Cortical Mandibular (ICM) proposto por Klemetti et al. ${ }^{17} \mathrm{em}$ imagens de reconstruções panorâmicas e transversais de tomografia computadorizada de feixe cônico. Os observadores avaliaram a cortical inferior das reconstruções panorâmicas e das imagens transaxiais para classificar em C1 (margem endosteal do córtex nítida), C2 (margem endosteal que apresentou defeitos semilunares) e $\mathrm{C}_{3}$ (camada cortical com pesados resíduos corticais e claramente porosos). Os resultados mostraram que não houve diferenças estatisticamente significantes entre utilizar a reconstrução panorâmica ou os cortes transaxiais, concluindo que imagens de TCFC podem auxiliar na identificação de pacientes com baixa massa óssea e encaminhá-los para os demais exames e tratamento.

Barngkgei et al. ${ }^{16}$ avaliaram a estrutura de osso trabecular de mandíbulas e a densidade - no processo odontóide da segunda vértebra cervical - entre mulheres com osteoporose e mulheres não osteoporóticas, por meio da utilização da TCFC. Os parâmetros estudados foram espessura trabecular (Tb.Th), a separação trabecular (Tb.S), fração de 
volume ósseo (BV/TV), superfície óssea específica (BS/TV) e densidade de conectividade. Os autores concluíram que a estrutura óssea trabecular da mandíbula e da maxila, avaliada pela TCFC, não é afetada pela osteoporose. Já a análise da densidade do osso trabecular revelou o oposto, mostrando que algumas medidas do osso trabecular podem ser avaliadas pela TCFC e que essas mensurações podem ajudar na previsão de osteoporose.

\section{DISCUSSÃO}

O número de estudos que avaliaram TCFC e osteoporose sem abordar unidades Hounsfield é muito pequeno., ${ }^{1,16,20,21} \mathrm{O}$ diagnóstico precoce por meio de medidas de qualidade óssea permite a adequada gestão da osteoporose, diminuindo o risco de fraturas, de incapacitação, e evita a dor subsequente destes pacientes. ${ }^{15,16,21}$

A perda óssea ocorre com o avanço da idade em homens e mulheres (osteoporose relacionada com a idade). Entretanto, nas mulheres a taxa de perda óssea também aumenta com a chegada da menopausa (osteoporose pós-menopausa), ${ }^{12}$ e esta é a razão pela qual os estudos selecionados foram com a população feminina.

Koh e Kim ${ }^{1}$ e Gomes et al. ${ }^{21}$ mostraram que o ICM avaliado em imagens de radiografias panorâmicas são comparáveis aos dos cortes tomográficos, validando sua utilização em TCFC.

A concordância intraobservador do estudo de Koh e Kim ${ }^{1}$ mostrou uma correlação significativa entre os ITC e os grupos normais e osteoporóticos. Os autores não realizaram o teste interobservador, porque apenas um radiologista treinado poderia realizar as leituras das imagens. Ainda que as leituras em dois tempos diferentes tenham sido realizadas, é importante que haja mais de um observador treinado, para que o método possa ser validado.

O estudo de Gomes et al. ${ }^{21}$ conclui que não houve diferenças estatisticamente significantes entre avaliar o índice em cortes de reconstrução panorâmica ou cortes transaxiais. Apesar dos autores relatarem que a TCFC pode ser utilizada como ferramenta de rastreio da osteoporose, os autores não correlacionaram seus resultados com a DEXA. Como a OMS preconiza o DEXA como o exame padrão-ouro para avaliação da qualidade óssea e diagnóstico da osteoporose, os novos métodos que estão sendo propostos pela literatura precisam apresentar uma correlação significativa com o padrão-ouro para que possam ser implementados como ferramentas opcionais de diagnósticos.

Diniz-Freitas et al. ${ }^{20}$ validou o IM de Taguchi et al. ${ }^{18}$ em cortes transaxiais da TCFC de pacientes que utilizavam bifosfonatos e do grupo controle, relatando em seu estudo que a espessura do trabeculado ósseo era maior nas mulheres que utilizavam o medicamento - diferentemente dos estudos realizados com radiografias panorâmicas, ${ }^{18}$ que mostravam que o IM é menor em mulheres com baixa densidade óssea comparadas com pacientes saudáveis.

O estudo de Barngkgei et al. ${ }^{16}$ mostrou que a análise da densidade do osso trabecular pode ajudar na previsão da osteoporose. Porém, como os autores usaram como parâmetros dados utilizados em micro-TC (BV/TV, BS/TV, Tb,Th, Tb.Sp, densidade trabecular), e os estudos prévios sobre osteoporose e TCFC utilizam os índices radiomorfométricos, a discussão desses resultados se tornou difícil.

Como os estudos citados apresentam diferentes metodologias, torna-se difícil comparar os resultados dos estudos, sendo necessária uma maior padronização de métodos e parâmetros. Atualmente, o uso de exames de TCFC permite a visualização das estruturas, sem sobreposição, ampliação ou distorção, além de visualização tridimensional do volume ósseo e arquitetura. ${ }^{21,22}$ Os softwares utilizados para avaliação das imagens de TCFC possuem diferentes ferramentas que auxiliam e permitem a obtenção dos índices radiomorfométricos.

Quando se sugerem novos métodos de diagnóstico, é necessário pensar em custo e benefício para 
o paciente. A DEXA, em comparação com a TCFC e a radiografia panorâmica, apresenta o maior custo, porém a menor dose de radiação. ${ }^{14}$ Neste estudo não foi avaliada a questão da dose de radiação, mas é importante ressaltar que o princípio ALARA (as low as reasonably achievable) deve ser sempre aplicado. ${ }^{7}$

\section{CONCLUSÃO}

Esta revisão de literatura demonstra que existe uma grande variabilidade de parâmetros e metodologias, e que estes não são conclusivos quanto à utilização da TCFC como método de rastreio da osteoporose. Pode-se afirmar que mais estudos nessa área precisam ser realizados para verificar a aplicabilidade, a especificidade e a sensibilidade da utilização desse método.

\section{REFERÊNCIAS}

1. Koh KJ, Kim KA. Utility of the computed tomography indices on cone beam computed tomography images in the diagnosis of osteoporosis in women. Imaging Sci Dent. 2011 Sep;41(3):101-6. doi: 10.5624/isd.2011.41.3.101.

2. Valerio CS, Trindade AM, Mazzieiro ET, Amaral TP, Manzi FR. Use of digital panoramic radiography as an auxiliary means of low bone mineral density detection in post-menopausal women. Dentomaxillofac Radiol. 2013;42(10):20120059. doi: 10.1259/dmfr.20120059.

3. Tosoni GM, Lurie AG, Cowan AE, Burleson JA. Pixel intensity and fractal analyses: detecting osteoporosis in perimenopausal and postmenopausal women by using digital panoramic images. Oral Surg Oral Med Oral Pathol Oral Radiol Endod. 2006 Aug;102(2):235-41. doi: http://dx.doi. org/10.1016/j.tripleo.2005.08.020.

4. Hua Y, Nackaerts O, Duyck J, Maes F, Jacobs R. Bone quality assessment based on cone beam computed tomography imaging. Clin Oral Implants Res. 2009 Aug;20(8):767-71. doi: 10.1111/j.1600-0501.2008.01677.x.

5. Homolka P, Beer A, Birkfellner W, Nowotny R, Gahleitner A, Tschabitscher M, et al. Bone mineral density measurement with dental quantitative CT prior to dental implant placement in cadaver mandibles: pilot study. Radiology. 2002 Jul;224(1):247-52. doi: http://dx.doi.org/10.1148/radiol.2241010948.
6. Mozzo P, Procacci C, Tacconi A, Martini PT, Andreis IA. A new volumetric CT machine for dental imaging based on the cone-beam technique: preliminary results. Eur Radiol. 1998;8(9):1558-64. doi:10.1007/s003300050586.

7. Spin-Neto R, Gotfredsen E, Wenzel A. Impact of voxel size variation on CBCT-based diagnostic outcome in dentistry: a systematic review. J Digit Imaging. 2013 Aug;26(4):813-20. doi: 10.1007/s10278-012-9562-7.

8. Tozoğlu U, Cakur B. Evaluation of the morphological changes in the mandible for dentate and totally edentate elderly population using cone-beam computed tomography. Surg Radiol Anat. 2014 Sep;36(7):643-9. doi: 10.1007/soo276013-1241-y.

9. Buttros DA, Nahas-Neto J, Nahas EA, Cangussu LM, Barral AB, Kawakami MS. Fatores de risco para osteoporose em mulheres na pós-menopausa do sudeste brasileiro. Rev Bras Ginecol Obstet. 2011 Jun;33(6):295-302. doi: http://dx.doi. org/10.1590/So100-72032011000600006.

10. López-López J, Estrugo-Devesa A, Jane-Salas E, AyusoMontero R, Gómez-Vaquero C. Early diagnosis of osteoporosis by means of orthopantomograms and oral x-rays: a systematic review. Med Oral Patol Oral Cir Bucal. 2011 Nov;16(7):e905-13. doi:10.4317/medoral.17304.

11. Benson BW, Prihoda TJ, Glass BJ. Variations in adult cortical bone mass as measured by a panoramic mandibular index. Oral Surg Oral Med Oral Pathol. 1991 Mar;71(3):34956. doi: 10.1016/o030-4220(91)90314-3. doi:10.1016/oo304220(91)90314-3.

12. Horner K, Devlin H. The relationship between mandibular bone mineral density and panoramic radiographic measurements. J Dent. 1998 May;26(4):337-43. doi: 10.1016/So3oo5712(97)0oo20-1. 10.1016/So300-5712(97)00020-1.

13. Devlin H, Allen P, Graham J, Jacobs R, Nicopoulou-Karayianni K, Lindh $\mathrm{C}$, et al. The role of the dental surgeon in detecting osteoporosis: the OSTEODENT study. Br Dent J. 2008 May;204(10):E16; discussion 560-1. doi: 10.1038/ sj.bdj.2008.317.

14. Sheahan NF, Dowling A, O'Reilly G, Malone JF. Commissioning and quality assurance protocol for dual energy X-ray absorptiometry (DEXA) systems. Radiat Prot Dosimetry. 2005;117(1-3):288-90. doi: 10.1093/rpd/nci741.

15. Verheij JG, Geraets WG, van der Stelt PF, Horner K, Lindh C, Nicopoulou-Karayianni K, et al. Prediction of osteoporosis with dental radiographs and age. Dentomaxillofac Radiol. 2009 Oct;38(7):431-7. doi: 10.1259/dmfr/55502190. 
16. Barngkgei I, Joury E, Jawad A. An innovative approach in osteoporosis opportunistic screening by the dental practitioner: the use of cervical vertebrae and cone beam computed tomography with its viewer program. Oral Surg Oral Med Oral Pathol Oral Radiol. 2015 Nov;120(5):651-9. doi: 10.1016/j.00oo.2015.08.008.

17. Klemetti E, Kolmakov S, Kröger H. Pantomography in assessment of the osteoporosis risk group. Scand J Dent Res. 1994 Feb;102(1):68-72. doi: 10.1111/j.16oo-0722.1994.tbo1156.x.

18. Taguchi A, Tanimoto K, Suei Y, Otani K, Wada T. Oral signs as indicators of possible osteoporosis in elderly women. Oral Surg Oral Med Oral Pathol Oral Radiol Endod. 1995 Nov;8o(5):612-6. doi: http://dx.doi.org/10.1016/S10792104(05)80158-1 10.1016/S1079-2104(05)80158-1.

19. Devlin H, Horner K. Mandibular radiomorphometric indices in the diagnosis of reduced skeletal bone mineral density. Osteoporos Int. 2002 May;13(5):373-8. doi: 10.1007/ so01980200042.

20. Diniz-Freitas M, Fernández-Montenegro P, Fernández-Feijoo J, Limeres-Posse J, González-Mosquera A, Vázquez-García E, et al. Mandibular cortical indices on cone-beam computed tomography images in osteoporotic women on treatment with oral bisphosphonates. Gerodontology. 2016 Jun;33(2):155-6o. doi: 10.1111/ger.12121. Epub 2014 Apr 4.
21. Gomes CC, de Rezende Barbosa GL, Bello RP, Bóscolo FN, de Almeida SM. A comparison of the mandibular index on panoramic and cross-sectional images from CBCT exams from osteoporosis risk group. Osteoporos Int. 2014 Jul;25(7):188590. doi: 10.1007/soo198-014-2696-3.

22. Scarfe WC, Farman AG, Sukovic P. Clinical applications of cone-beam computed tomography in dental practice. J Can Dent Assoc. 2006 Feb;72(1):75-80.

23. Scarfe WC, Levin MD, Gane D, Farman AG. Use of cone beam computed tomography in endodontics. Int $\mathrm{J}$ Dent. 2009;2009:634567. doi: 10.1155/2009/634567.

24. De Vos W, Casselman J, Swennen GR. Cone-beam computerized tomography (CBCT) imaging of the oral and maxillofacial region: a systematic review of the literature. Int $\mathrm{J}$ Oral Maxillofac Surg. 2009 Jun;38(6):609-25. doi: 10.1016/j. ijom.2009.02.028.

25. Kamburoğlu K, Murat S, Kolsuz E, Kurt H, Yüksel S, Paksoy C. Comparative assessment of subjective image quality of cross-sectional cone-beam computed tomography scans. J Oral Sci. 2011 Dec;53(4):501-8. doi: http://doi.org/10.2334/ josnusd.53.501.

26. Hatcher DC. Operational principles for cone-beam computed tomography. J Am Dent Assoc. 2010 Oct;141 Suppl 3:3S-6S. doi: http://dx.doi.org/10.14219/jada.archive.2010.0359 